10

\title{
Влияние сильной низкочастотной волны на распространение слабых ультразвуковых импульсов в стержневом резонаторе из отожженной поликристаллической меди
}

\author{
(C) В.Е. Назаров, ${ }^{1}$ А.Б. Колпаков ${ }^{2}$ \\ ${ }^{1}$ Федеральный исследовательский центр Институт прикладной ффизики РАН, \\ 603950 Нижний Новгород, Россия \\ ${ }^{2}$ Нижегородский государственный университет им. Н.И. Лобачевского, \\ 603022 Нижний Новгород, Россия \\ e-mail: v.e.nazarov@appl.sci-nnov.ru
}

Поступило в Редакцию 5 октября 2021 г.

В окончательной редакции 12 января 2022 г.

Принято к публикации 19 января 2022 г.

\begin{abstract}
Проведено экспериментальное исследование влияния сильной низкочастотной волны на распространение слабых ультразвуковых импульсов в акустическом резонаторе из отожженной поликристаллической меди. Измерения проводились при гармоническом возбуждении резонатора на его первых четырех продольных модах в диапазоне от 2 до $15 \mathrm{kHz}$, частота ультразвуковых импульсов изменялась от 65 до $400 \mathrm{kHz}$. Анализ наблюдаемых нелинейных эффектов проведен в рамках уравнения состояния поликристалла, полученного на основе модификации струнной модели дислокации Гранато-Люкке. Определены значения параметров диссипативной и реактивной нелинейности дислокаций для отожженной меди.
\end{abstract}

Ключевые слова: дислокационная диссипативная и реактивная нелинейности, поликристаллическая медь, упругие волны.

DOI: $10.21883 / J T F .2022 .04 .52251 .266-21$

\section{Введение}

К актуальным вопросам современной акустики относятся задачи, связанные с изучением нелинейных волновых процессов (НВП) в микронеоднородных твердых телах [1-3], обладающих аномально высокой неаналитической и частотнозависимой нелинейностью. Актуальность этих вопросов обусловлена тем, что „классическая“ пятиконстантная теория упругости $[4,5]$, отвечающая слабонелинейным однородным средам (с аналитической и частотнонезависимой нелинейностью), не объясняет закономерностей нелинейных акустических эффектов (НАЭ) в экспериментах с сильно нелинейными микронеоднородными средами, а „общей“ (универсальной) теории НВП для таких сред не существует.

К сильно нелинейным микронеоднородным твердотельным средам относятся многие поликристаллические металлы и горные породы. Их нелинейность связывается с движением и взаимодействием дислокаций (линейных дефектов поликристаллов) с точечными дефектами (вакансиями, примесными и межузельными атомами) [615]. В низкочастотном (НЧ) диапазоне поликристаллы проявляют гистерезисную нелинейность, а в высокочастотном (ВЧ) - диссипативную и реактивную [3]. Закономерности НАЭ, обусловленные гистерезисной, диссипативной и реактивной нелинейностями, как правило, различны, что создает возможность раздельного изучения вкладов этих нелинейностей в проявления НЧ и ВЧ нелинейных акустических эффектов.
Наиболее сильные проявления нелинейных свойств среды имеют место при относительно высоких амплитудах упругих волн, что легко реализуется в высокодобротных резонаторах. В резонаторах из поликристаллических твердых тел наблюдаются гистерезисные эффекты амплитудно-зависимого внутреннего трения нелинейные потери, сдвиг резонансных частот и генерация высших гармоник, а также эффекты затухания и фазовой задержки несущей (ФЗН) слабых ультразвуковых импульсов в поле мощной НЧ волны, обусловленные не НЧ гистерезисной, а ВЧ диссипативной и реактивной нелинейностью $[3,16]$.

В различных средах нелинейные эффекты часто проявляются по-разному, поэтому установление амплитудночастотных зависимостей НАЭ в микронеоднородных средах является одной из основных задач при проведении их экспериментальных исследований. Фундаментальная цель таких исследований связывается с выявлением механизмов аномально высокой акустической нелинейности различных микронеоднородных сред. Прикладной же направленностью таких работ является создание и развитие нелинейных акустических методов диагностики микронеоднородных сред и материалов $[3,17,18]$.

В настоящей работе, являющейся продолжением работы [19], представлены новые результаты экспериментальных исследований влияния сильной НЧ волны накачки в стержневом резонаторе из отожженной поликристаллической меди на распространение в нем слабых ультразвуковых импульсов (УИ). Установлены 


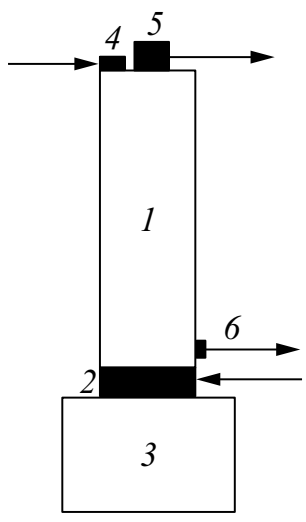

Рис. 1. Схема эксперимента.

амплитудно-частотные зависимости нелинейных затухания и ФЗН ультразвуковых импульсов под действием сильной низкочастотной волны. Анализ наблюдаемых нелинейных эффектов (затухания и ФЗН ультразвуковых импульсов) проведен в рамках уравнения состояния поликристалла, содержащего дислокационные диссипативную и реактивную нелинейности [20]. Из сравнения экспериментальных и аналитических результатов определены значения параметров диссипативной и реактивной нелинейности дислокаций для отожженной меди.

\section{1. Схема эксперимента}

Эксперименты по изучению влияния сильной НЧ стоячей волны на распространение слабых бегущих УИ проводились со стержневым резонатором, изготовленным из отожженной меди. Длина стержня $L=0.43 \mathrm{~m}$. Температура отжига $-600^{\circ} \mathrm{C}$, время отжига - около 2h. (В предыдущей работе [19] в том же резонаторе исследовались НЧ эффекты амплитудно-зависимого внутреннего трения, возникающие из-за гистерезисной нелинейности отожженной меди при НЧ гармоническом возбуждении резонатора.)

Схема экспериментальной установки приведена на рис. 1. К нижнему торцу стержня 1 , для возбуждения в нем продольной НЧ волны накачки, приклеивался пьезокерамический излучатель 2, другая его сторона была приклеена к массивной нагрузке 3 . К верхнему свободному торцу стержня 1 приклеивались ВЧ пьезокерамический излучатель 4 (для излучения продольных УИ, распространяющихся вдоль оси стержня) и пьезоприемник 5 (для приема НЧ колебаний, создаваемых излучателем 2). Вблизи излучателя 2 на боковой поверхности стержня 1 приклеивался пьезоприемник 6 , который реагировал на продольные ВЧ колебания стержня (вдоль его оси). При помощи пьезоприемника 6 осуществлялся прием и измерение амплитуды и ФЗН, прошедших через стержень УИ, создаваемых излучателем 4. Частота $f$ импульсов изменялась в диапазоне от 65 до $400 \mathrm{kHz}$.

В этом эксперименте для НЧ волны стержень 1 являлся резонатором, а для бегущих УИ - фактически безграничной средой. Собственные частоты $F_{p}$
НЧ продольных мод такого резонатора определяются выражением: $F_{p} \cong(2 p-1) C_{0} / 4 L, C_{0}-$ фазовая скорость НЧ продольной волны в стержне, $p$ - номер моды, $p=1,2,3,4 \ldots$ Для первых четырех продольных мод резонатора его резонансные частоты $F_{p}$ и добротности $Q_{p}$ составляли соответственно: $F_{1}=2204 \mathrm{~Hz}$, $F_{2}=6447 \mathrm{~Hz}, F_{3}=10697 \mathrm{~Hz}, F_{4}=14928 \mathrm{~Hz}$ и $Q_{1}=450$, $Q_{2}=883, Q_{3}=578, Q_{4}=711$.

\section{2. Результаты измерений}

В эксперименте в стержне 1 создавались сильная НЧ стоячая волна накачки и слабые УИ. Прошедшие через стержень импульсы принимались пьезоприемником 6 и поступали на осциллограф, где производились измерения их амплитуды $U\left(\varepsilon_{m}\right)$ и $Ф 3 Н ~ \Delta \tau\left(\varepsilon_{m}\right)$ в зависимости от амплитуды деформации $\varepsilon_{m}$ НЧ волны (в резонансе).
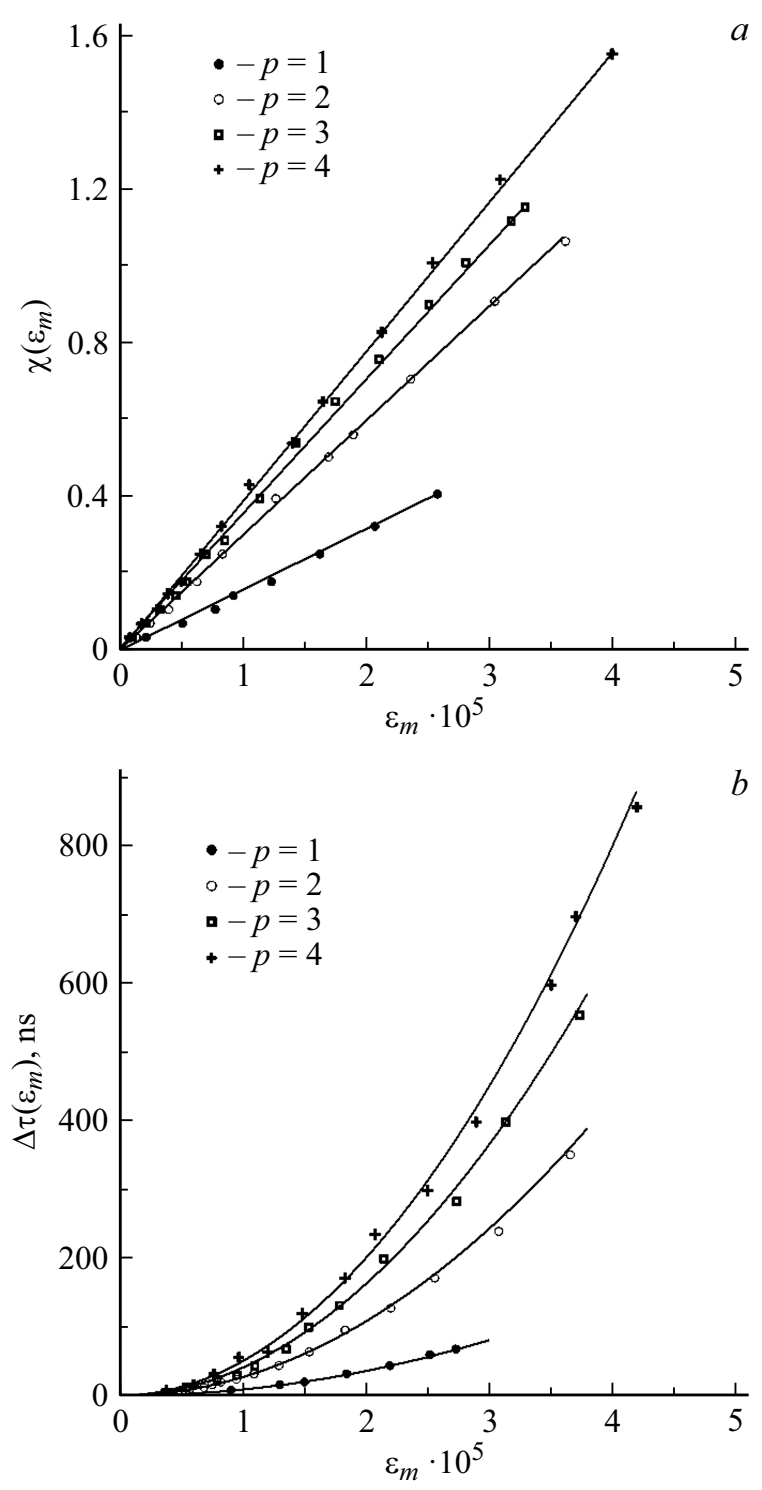

Рис. 2. Результаты измерений зависимостей $\chi\left(\varepsilon_{m}\right)(a)$ и $\Delta \tau\left(\varepsilon_{m}\right) \quad(b)$ от $\varepsilon_{m}$ при $f=365 \mathrm{kHz}$. Линии соответствуют зависимостям: $\chi\left(\varepsilon_{m}\right) \propto \varepsilon_{m}, \Delta \tau\left(\varepsilon_{m}\right) \propto \varepsilon_{m}^{2}$. 
При увеличении $\varepsilon_{m}$ амплитуда $U\left(\varepsilon_{m}\right)$ принимаемых УИ уменьшалась, а их ФЗН $\Delta \tau\left(\varepsilon_{m}\right)$ увеличивалась, т.е. затухание импульсов увеличивалось, а фазовая скорость уменьшалась.

На рис. 2 приведены зависимости коэффициента нелинейного затухания $\chi\left(\varepsilon_{m}\right)=\ln \left[U_{0} / U\left(\varepsilon_{m}\right)\right]\left(U_{0}\right.$ - амплитуда импульсов при $\left.\varepsilon_{m}=0\right)$ и фазовой задержки несущей $\Delta \tau\left(\varepsilon_{m}\right)$ УИ с частотой $f=365 \mathrm{kHz}$ от амплитуды $\varepsilon_{m}$ НЧ волны при $p=1,2,3,4$. Из рис. 2 видно, что имеют место следующие зависимости: $\chi\left(\varepsilon_{m}\right) \propto \varepsilon_{m}, \Delta \tau\left(\varepsilon_{m}\right) \propto \varepsilon_{m}^{2}$, при этом $\chi\left(\varepsilon_{m}\right)$ и $\Delta \tau\left(\varepsilon_{m}\right)$ при $\varepsilon_{m}=$ const растут с ростом частоты $F_{p}$ НЧ волны.

На рис. 3 приведены зависимости коэффициента $\chi\left(\varepsilon_{m}\right)$ и фазовой задержки $\Delta \tau\left(\varepsilon_{m}\right)$ УИ с частотами $f=365 \mathrm{kHz}$ от частоты $F_{p}$ при $\varepsilon_{m}=2 \cdot 10^{-5}$. Из этих рисунков видно,

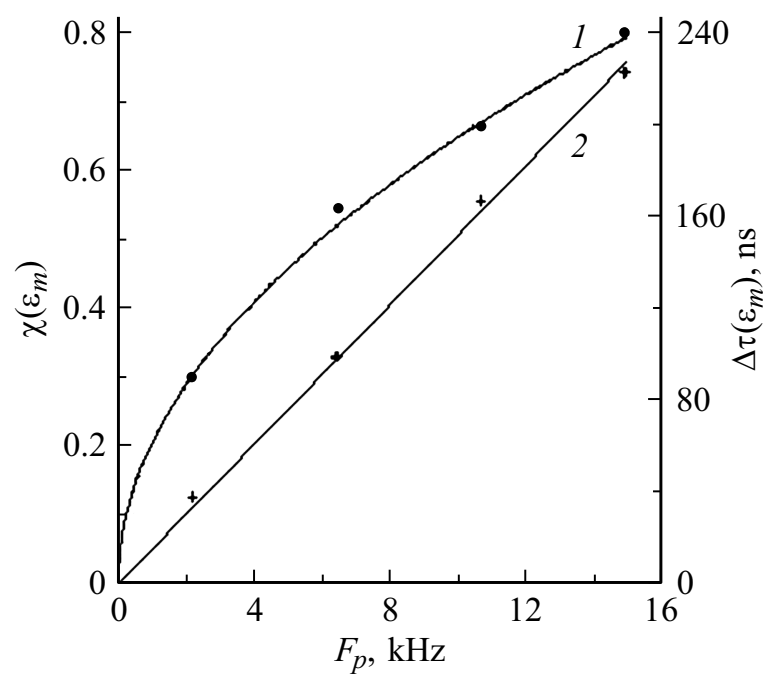

Рис. 3. Результаты измерений зависимостей $\chi\left(\varepsilon_{m}\right)$ (1) и $\Delta \tau\left(\varepsilon_{m}\right)(2)$ от $F_{p}$ при $\varepsilon_{m}=2 \cdot 10^{-5}$ и $f=365 \mathrm{kHz}$. Линии соответствуют зависимостям: $1-\chi\left(\varepsilon_{m}\right) \propto F_{p}^{1 / 2}, 2-\Delta \tau\left(\varepsilon_{m}\right) \propto F_{p}$.

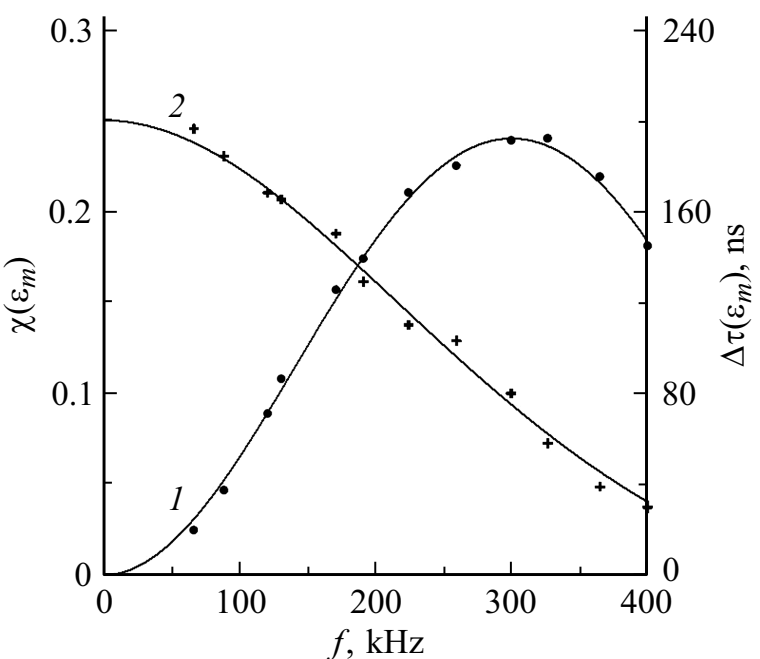

Рис. 4. Зависимости $\chi\left(\varepsilon_{m}\right)(1)$ и $\Delta \tau\left(\varepsilon_{m}\right)$ 2) от частоты $f$ при возбуждении резонатора на частоте второй моды $(p=2)$ при $\varepsilon_{m}=6.8 \cdot 10^{-6}$. Линии 1 и $2-$ результат расчета по формулам (1), (2); • и + - результаты измерений. что $\chi\left(\varepsilon_{m}\right) \propto F_{p}^{1 / 2}, \Delta \tau\left(\varepsilon_{m}\right) \propto F_{P}$. Следует отметить, что зависимости $\chi=\chi\left(\varepsilon_{m}\right)$ и $\Delta \tau=\Delta \tau\left(\varepsilon_{m}\right)$ от $\varepsilon_{m}$ и $F_{p}$ не соответствуют ни амплитудным, ни частотным зависимостям НЧ эффектов амплитудно-зависимого внутреннего трения, установленных в работе [19] для того же резонатора и обусловленных гистерезисной нелинейностью отожженной меди.

На рис. 4 приведены зависимости коэффициента затухания $\chi=\chi\left(\varepsilon_{m}\right)$ и ФЗН $\Delta \tau=\Delta \tau\left(\varepsilon_{m}\right)$ от частоты импульса $f$ при $\varepsilon_{m}=6.8 \cdot 10^{-6}$ и $p=2$. Из рис. 4 следует, что с ростом частоты $f$ коэффициент $\chi=\chi\left(\varepsilon_{m}\right)$ вначале (при $65 \mathrm{kHz}<f<300 \mathrm{kHz}$ ) растет, а затем (при $300 \mathrm{kHz}<f<400 \mathrm{kHz}$ ) падает, а задержка $\Delta \tau\left(\varepsilon_{m}\right)$ при $65 \mathrm{kHz}<f<400 \mathrm{kHz}$ падает.

\section{3. Анализ и сравнение экспериментальных и теоретических результатов}

Теоретическое описание наблюдаемых в эксперименте эффектов приведено в работе [20], где в рамках уравнения состояния поликристаллического твердого тела с дислокационной диссипативной и реактивной нелинейностью получены выражения для $\chi=\chi\left(\varepsilon_{m}\right)$ и $\Delta \tau\left(\varepsilon_{m}\right)$ :

$$
\begin{aligned}
& \chi\left(\varepsilon_{m}\right)=\frac{\mu P}{\sqrt{\pi}} \frac{\Gamma[(m+1) / 2]}{\Gamma[(m+2) / 2]} \varepsilon_{m}^{m} L \Omega_{P}^{q} d_{0} \omega^{2} \\
& \times \int_{0}^{\infty} \frac{\left[\left(\Omega^{2}-\omega^{2}\right)^{2}-d_{0}^{2} \omega^{2}\right] l N(l) d l}{\left[\left(\Omega^{2}-\Omega_{p}^{2}\right)^{2}+d_{0}^{2} \Omega_{p}^{2}\right]^{m / 2}\left[\left(\Omega^{2}-\omega^{2}\right)^{2}+d_{0}^{2} \omega^{2}\right]^{2}}, \\
& \tau\left(\varepsilon_{m}\right)=\frac{\eta Q}{\sqrt{\pi}} \frac{\Gamma[(n+1) / 2]}{\Gamma[(n+2) / 2]} \varepsilon_{m}^{n} L \Omega_{P}^{r} \\
& \times \int_{0}^{\infty} \frac{\left[\left(\Omega^{2}-\omega^{2}\right)^{2}-d_{0}^{2} \omega^{2}\right] \Omega^{2} l N(l) d l}{\left[\left(\Omega^{2}-\Omega_{p}^{2}\right)^{2}+d_{0}^{2} \Omega_{p}^{2}\right]^{n / 2}\left[\left(\Omega^{2}-\omega^{2}\right)^{2}+d_{0}^{2} \omega^{2}\right]^{2}},
\end{aligned}
$$

где

$$
\begin{aligned}
P=\frac{8 R^{2} C_{0}}{\pi^{9 / 2}} & \frac{(1+q) \Gamma[(m+3) / 2]}{\Gamma[(m+4) / 2]} B\left[\frac{m-q+1}{2}, \frac{q+1}{2}\right] \\
& \times\left(\frac{4 R C_{0}^{2}}{\pi^{2} b^{2}}\right)^{m}\left(\frac{b}{C_{\perp}}\right)^{q},
\end{aligned}
$$

$$
\begin{aligned}
Q=\frac{8 R^{2} C_{0}}{\pi^{9 / 2}} & \frac{(1+n-r) \Gamma[(n+3) / 2]}{\Gamma[(n+4) / 2]} B\left[\frac{n-r+1}{2}, \frac{r+1}{2}\right] \\
& \times\left(\frac{4 R C_{0}^{2}}{\pi^{2} b^{2}}\right)^{n}\left(\frac{b}{C_{\perp}}\right)^{r},
\end{aligned}
$$

$G$ и $E=2 G /(1+v)$ - модуль сдвига и модуль Юнга; $C_{\perp}=(G / \rho)^{1 / 2}$ - скорость сдвиговой волны; $C_{0}=(E / \rho)^{1 / 2} ; v$ и $\rho-$ коэффициент Пуассона и плотность, $b-$ модуль вектора Бюргерса; $A=\pi \rho b^{2}-$ масса единицы длины дислокации, $B$ и $C=2 G b^{2} / \pi(1-v)-$ 
коэффициент линейного трения и коэффициент линейного натяжения дислокации; $l, \Omega(l)=[2 /(1-v)]^{1 / 2}\left(C_{\perp} / l\right)$ и $d_{0}=B / A-$ длина, резонансная частота и параметр демпфирования дислокации; $\mu, \eta$ и $m, q, n, r-$ безразмерные параметры и показатели степени диссипативной и реактивной нелинейности дислокации, $m \geq q \geq 0$, $n \geq r \geq 0, N(l)-$ функция распределения дислокаций по длинам $l, \int_{0}^{\infty} l N(l) d l=\Lambda, \Lambda$ - плотность дислокаций, $R$ - ориентационный фактор, $\Omega_{p}=2 \pi F_{P}, \omega=2 \pi f$.

Проведем анализ экспериментальных и теоретических результатов и определим параметры дислокационной структуры отожженной меди.

Из сравнения выражений (1), (2) с результатами измерений (рис. 2,3) сразу следует, что $m=1, q=1 / 2$, $n=2, r=1$.

Подберем теперь функцию распределения $N(l)$ и параметры дислокаций, так чтобы получилось соответствие зависимостей (1), (2) от частоты $f=\omega / 2 \pi$ с результатами измерений (рис. 4). Хорошее соответствие имеет место для модифицированной функции распределения Келера

$$
N(l)=\frac{\Lambda \exp \left[-\left(l-l_{0}\right) / L_{0}\right]}{L_{0}\left(l_{0}+L_{0}\right)}, 0 \leq l_{0} \leq l<\infty,
$$

при следующих параметрах: $C_{\perp}=2.3 \cdot 10^{3} \mathrm{~m} / \mathrm{s}, C_{0}=$ $3.8 \cdot 10^{3} \mathrm{~m} / \mathrm{s}, v=0.28, \Lambda=10^{12} \mathrm{~m}^{-2}, L_{0}=10^{-4} \mathrm{~m}, l_{0}=$ $5 \cdot 10^{-5} \mathrm{~m}, b=3 \cdot 10^{-10} \mathrm{~m}, d_{0}=10^{9} \mathrm{~s}^{-1}, R^{3}=7.7 \cdot 10^{-3}$, $R^{4}=3.03 \cdot 10^{-3}, \mu=4.1 \cdot 10^{-1}, \eta=1.5 \cdot 10^{2}$.

Для функции распределения Келера [6], полученной для случая беспорядочного расположения примесных атомов вдоль дислокационной линии, длина $l_{0}=0$, при этом $L_{0}=\int_{0}^{\infty} l N(l) d l / \int_{0}^{\infty} N(l) d l-$ это средняя длина дислокации. Для модифицированного распределения Келера (3) $-l_{0}>0\left(l_{0} \gg b\right)$, а средняя длина дислокации $\langle l\rangle$ определяется выражением $\langle l\rangle=L_{0}+l_{0}$.

\section{Заключение}

Приведены результаты исследований эффектов влияния сильной НЧ волны на затухание и фазовую задержку несущей слабых УИ в стержневом резонаторе из отожженной поликристаллической меди и показано, что ее акустическая нелинейность содержит диссипативную и реактивную составляющие. Из сравнения экспериментально установленных амплитудно-частотных зависимостей с теоретическими [20], полученными на основе модификации струнной модели дислокации ГранатоЛюкке, определены основные характеристики дислокаций отожженной меди (функция распределения дислокаций по длинам, параметр демпфирования, показатели степеней и параметры диссипативной и реактивной нелинейности). Из анализа результатов, полученных здесь и в работе [19], следует, что проявления гистерезисной, диссипативной и реактивной нелинейностей отожженной меди качественно отличаются, поскольку амплитудные и частотные зависимости НЧ и ВЧ нелинейных эффектов различны. Это свидетельствует о том, что механизмы гистерезисной и диссипативной и реактивной нелинейностей отожженной меди также различны. Гистерезисная нелинейность поликристаллических твердых тел связана с периодическим отрывом дислокаций от примесных атомов (и закреплением на них же), а диссипативная и реактивная нелинейности с нелинейными трением и натяжением дислокаций, совершающих колебательное движение под действием интенсивной упругой волны в окружении точечных дефектов поликристалла. Результаты проведенных исследований свидетельствуют о том, что дислокационные гистерезисная, диссипативная и реактивная нелинейности являются чувствительными характеристиками поликристаллических твердых тел, что можно использовать для их нелинейной акустической диагностики.

\section{Финансирование работы}

Работа поддержана РФФИ (грант N20-02-00215A).

\section{Конфликт интересов}

Авторы заявляют, что у них нет конфликта интересов.

\section{Список литературы}

[1] М.А. Исакович. Общая акустика (Наука, М., 1973) [M.A. Isakovich. General Acoustics (Cambrige, 1973)]

[2] R.A. Guyer, P.A. Johnson. Nonlinear Mesoscopic Elasticity: the Complex Behaviour of Granular Media Including Rocks and Soil (Wiley-VCH Verlag GmbH \& Co. KgaA, Weinheim, 2009)

[3] V.E. Nazarov, A.V. Radostin. Nonlinear Acoustic Waves in Micro-Inhomogeneous Solids (John Wiley \& Sons, Chichester, 2015)

[4] Л.К. Зарембо, В.А. Красильников. УФН, $102(4), 549$ (1970).

[5] Л.Д. Ландау, Е.М. Лифшиц. Теория упругости (Наука, М., 1987)

[6] A. Granato, K. Lucke. J. Appl. Phys., 27 (5), 583 (1956).

[7] Ультразвуковые методы исследования дислокаций: Сб. статей. Пер. с англ. и нем. под ред. Л.Г. Меркулова (ИИЛ, М., 1963)

[8] Application to Quantum and Solid State Physics, in Physical Acoustics: Principles and Methods. Ed. by Warren P. Mason (Academic Press, NY. and London, 1966), v. 4, part A.

[9] The Effect of Imperfection, in Physical Acoustics: Principles and Methods. Ed. by Warren P. Mason (Academic Press, NY. and London, 1966), v. 3, part A.

[10] Д. Ниблетт, Дж. Уилкс. УФН, 80 (1), 125 (1963).

[11] R. Truell, C. Elbaum, B.B. Chick. Ultrasonic Methods in Solid State Physics (Academic Press, NY. and London, 1969)

[12] В.П. Левин, В.Б. Проскурин. Дислокационная неупругость в металлах (Наука, М., 1993)

[13] T. Suzuki. Dislocation Dynamics (McGraw Hill, NY., 1967/1968) 
[14] H.F. Pollard. Sound Waves in Solids (Pion Limited, 1977)

[15] И.И. Новиков, К.М. Розин. Кристаллография и дефекты кристаллической решетки (Металлургия, М., 1990)

[16] V.E. Nazarov, A.B. Kolpakov. Wave Motion, 72, 187 (2017). https://doi.org/10.1016/j.wavemoti.2017.03.003

[17] Nonlinear Ultrasonic and Vibro-Acoustical Techniques for Nondestructive Evaluation, ed. by T. Kundu (Cham: Springer Nature Swizerland AG, 2019), ISBN 978-3-319-94474-6

[18] C.J. Lissenden. J. Appl. Phys., 129, 021101 (2021). DOI: $10.1063 . / 5.0038340$

[19] В.Е. Назаров, А.Б. Колпаков. ЖТФ, 91 (9), 1305 (2021). DOI: $10.21883 /$ JTF.2021.09.51208.21-21

[20] В.Е. Назаров. ЖТФ, 90 (12), 2085 (2020).

DOI: 10.21883/JTF.2021.09.51208.21-21

[V.E. Nazarov. Tech. Phys., 65 (12), 1905 (2020).

DOI: $10.1134 / \mathrm{S} 1063784220120154]$ 